\title{
Knight moves: protect the pawns
}

\author{
Author: Carol Postlethwaite
}

\section{Aims}

$>$ To set up a Hospital at Night service.

> To protect the ward doctor and patients they review from the hazards of constant interruption by bleep.

\section{Methods}

Like many hospitals, East Surrey Hospital is as busy at night as it is during the day, but with far fewer staff to manage the workload. Specialty doctors worked for the most part in silos, with one meeting early in the shift gathering the site team and lead doctors in each specialty to establish bed and patient pressures during that shift. It was often poorly attended. The doctors covering the wards found it difficult to work efficiently and safely due to the high frequency of bleeps received that were interrupting patient reviews. Breaks were not possible, and the doctors were exhausted, had low morale, and were operating at high cognitive load, risking their and their patients' safety by the end of each shift.

Workstreams were initiated to:

$>$ quantify the bleep frequency per doctor on average

$>$ identify the frequent jobs being requested

$>$ gather leads for the specialties and nursing to create a working group to agree a new approach

$>$ create a resource for the ward staff to stream bleeps and jobs a new way

> develop a 2am huddle for refreshment, rest and discussion

$>$ upskill the clinical site management (CSM) team

> invest in information technology to support bleep-light working.

\section{Results}

The surgical senior house officer surprisingly had the highest frequency of bleeps closely followed by the medical registrar then the ward medic.

The ward jobs were audited by the medical ward doctor over 1 week and split into themes. These themes were then considered by the working group and split into doctor and non-doctor dependent jobs, which were reassigned.

An intranet and back-up paper resource was developed to guide the first call by the ward nurses, in the majority of cases to the CSM team who would then stream. The CSM team were upskilled, with simulation training developed to further support the role. Policy was signed off by all directorate leads.

We are waiting for a suitable package to allow smartphonedelivered jobs, to markedly reduce bleeps to emergency calls only.

\section{Conclusion}

Major changes to working require a long lead-in time to fully engage the participants. This leads to more successful change and the likelihood that it sticks. In our research we found old policies suggesting something similar had been attempted 10 years ago, which no one remembered.

\section{Conflict of interest statement}

None. 